\title{
Enhancement of the electric dipole moment of the electron in the YbF molecule*
}

\author{
M G Kozlov \\ Petersburg Nuclear Physics Institute, Gatchina, Leningrad district, 188350, Russia
}

\begin{abstract}
We calculate an effective electric field on the unpaired electron in the YbF molecule. This field determines sensitivity of the molecular experiment to the electric dipole moment of the electron. We use experimental value of the spin-doubling constant $\gamma$ to estimate the admixture of the configuration with the hole in the $4 \mathrm{f}$-shell of Ytterbium to the ground state of the molecule. This admixture reduces the field by $7 \%$. Our value for the effictive field is 5.1 a.u. $=2.5 \cdot 10^{10} \mathrm{~V} / \mathrm{cm}$.
\end{abstract}

\section{Introduction}

It is well known that effects caused by an electric dipole moment (EDM) of the electron $d_{\mathrm{e}}$ are strongly enhanced in heavy diatomic radicals (see, for example, a review [1]). An experimental search for the EDM of the electron is now underway in Brighton University on the YbF molecule [2]. For this reason the reliable calculations for this molecule are necessary. The existence of a shallow 4 f-shell adds complexity to such calculations. Here we extend the semiempirical approach suggested in [3, th to account for a possible admixture of the configuration with the hole in the 4 f-shell of Ytterbium. The idea that f-hole can explain small value of the spin-doubling constant $\gamma$ belongs to I. B. Khriplovich [5].

The EDM experiments with radicals are made on the spin-rotational levels of the electronic ground state. The spin-rotational degrees of freedom of the molecule are described by the following spin-rotational Hamiltonian [6 9]:

$$
H_{\mathrm{sr}}=B \mathbf{N}^{2}+\gamma \mathbf{S N}+\mathbf{S} \underline{\underline{\mathbf{A}}} \mathbf{I}+W_{d} d_{\mathrm{e}} \mathbf{S n} .
$$

In this expression $\mathbf{N}$ is the rotational angular momentum, $B$ is the rotational constant, $\mathbf{S}$ is the spin of the electron and $\mathbf{I}$ is the spin of the $\mathrm{Yb}$ nucleus, $\mathbf{n}$ is the unit vector directed along the molecular axis from $\mathrm{Yb}$ to $\mathrm{F}$. The spin-doubling constant $\gamma$ characterizes the spin-rotational interaction. The axial tensor $\underline{\underline{\mathbf{A}}}$ describes magnetic hyperfine structure. It can be determined by two parameters: $A=\left(A_{\|}+2 A_{\perp}\right) / 3$ and $A_{\mathrm{d}}=\left(A_{\|}-A_{\perp}\right) / 3$. The last term in (11) correspond to the interaction of the EDM of the electron $d_{\mathrm{e}}$ with the molecular field, $\frac{1}{2} W_{d}$ being the effective electric field on the electron.

Parameters $B, \gamma, A$ and $A_{\mathrm{d}}$ are known from the experiment [10 12]:

$$
B=7237 \mathrm{MHz}, \quad \gamma=13 \mathrm{MHz}, \quad A=7617 \mathrm{MHz}, \quad A_{\mathrm{d}}=102 \mathrm{MHz},
$$

while $W_{d}$ have to be calculated. There are three calculations of this parameter [4, 13, 14]. In earlier calculations [4, 13] 4f-shell of Ytterbium was frozen. In [14] f-electrons are included in the valence space, but details of this calculation are not yet published. Here we allow the admixture of the f-hole to the ground state of the molecule.

\section{Electronic wave function}

The ground state of $\mathrm{YbF}$ molecule is known to be $\Sigma_{1 / 2}$ [10]. The large hyperfine constants $A$ and $A_{\mathrm{d}}$ indicate that unpaired electron occupies molecular orbital $\sigma_{\mathrm{s}}$ with dominant contribution from 6s-orbital and significant contribution of $6 \mathrm{p}_{0}$-orbital of $\mathrm{Yb}$ ion. But a simple one-configurational wave function can not explain an exceptionally small value of the constant $\gamma$. Following Khriplovich we will assume that there is small admixture of the f-hole:

$$
|\Sigma, \omega\rangle=\left|\sigma_{\mathrm{s}}, \omega\right\rangle+\delta_{\mathrm{f}} \overline{\left|\sigma_{\mathrm{f}},-\omega\right\rangle}
$$

where $\omega= \pm \frac{1}{2}$ is the projection of the total electronic angular momentum on the molecular axis and the bar over the orbital corresponds to the hole. More explicitly (3) means that many electron wave function reads

${ }^{*}$ To be submitted to J. Phys. B 


$$
|\Sigma, \omega\rangle=[\ldots]\left(\left|\sigma_{\mathrm{f}},-\frac{1}{2}\right\rangle\left|\sigma_{\mathrm{f}}, \frac{1}{2}\right\rangle\left|\sigma_{\mathrm{s}}, \omega\right\rangle+\delta_{\mathrm{f}}\left|\sigma_{\mathrm{f}}, \omega\right\rangle\left|\sigma_{\mathrm{s}},-\frac{1}{2}\right\rangle\left|\sigma_{\mathrm{s}}, \frac{1}{2}\right\rangle\right)
$$

where [...] denotes the closed core. The spin-orbit interaction for the f-hole is large and has the opposite sign. This can explain the small value of $\gamma$ (see below).

All constants of the Hamiltonian (1) except for the constant $B$ depend only on the electron spin density in the vicinity of the heavy nucleus. For this reason we can expand molecular orbitals in spherical waves with the origin at the Yb nucleus:

$$
\begin{aligned}
\left|\sigma_{\mathrm{s}}, \omega\right\rangle & =\left(x_{\mathrm{s}}|\tilde{6} s\rangle+x_{\mathrm{p}}\left|\tilde{6} p_{0}\right\rangle+x_{\mathrm{d}}\left|\tilde{5} d_{0}\right\rangle+\ldots\right)|\omega\rangle \\
\left|\sigma_{\mathrm{f}}, \omega\right\rangle & =\left(\left|\tilde{4} f_{0}\right\rangle+\varepsilon_{\mathrm{d}}\left|\tilde{5} d_{0}+\ldots\right\rangle\right)|\omega\rangle
\end{aligned}
$$

where $|\omega\rangle$ denotes spin function and dots stand for the higher spherical waves. The radial functions in this expansion can be considered as distorted orbitals of the $\mathrm{Yb}^{+}$ion. Corresponding principle quantum numbers are marked with tilde.

Wave function (3) is written in a pure nonrelativistic coupling case. Spin-orbit interaction $H_{\text {so }}$ mixes state (3) with $\Pi_{1 / 2}$ states. One can expect that the largest admixtures correspond to the molecular orbitals $\pi_{\mathrm{p}}$ and $\pi_{\mathrm{f}}$ (the spin-orbit interaction for the $5 \mathrm{~d}$-shell is much smaller):

$$
\begin{aligned}
\left|\pi_{\mathrm{p}}, \omega\right\rangle & =\left(a_{\mathrm{p}}\left|\tilde{6} p_{2 \omega}\right\rangle+\ldots\right)|-\omega\rangle, \\
\left|\pi_{\mathrm{f}}, \omega\right\rangle & =\left(\left|\tilde{4} f_{2 \omega}\right\rangle+\ldots\right)|-\omega\rangle .
\end{aligned}
$$

Then, the wave function of the ground state has the form

$$
|\Sigma, \omega\rangle=\left|\sigma_{\mathrm{s}}, \omega\right\rangle+c_{\mathrm{p}}\left|\pi_{\mathrm{p}}, \omega\right\rangle+\delta_{\mathrm{f}} \overline{\left|\sigma_{\mathrm{f}},-\omega\right\rangle}+c_{\mathrm{f}} \overline{\left|\pi_{\mathrm{f}},-\omega\right\rangle} .
$$

\section{Hyperfine tensor and parameter $W_{d}$}

The operator of the hyperfine interaction in atomic units has the form

$$
H_{\mathrm{hf}}=\frac{g_{\mathrm{n}} \alpha}{2 m_{\mathrm{p}}}(\vec{\alpha} \times \mathbf{r} \cdot \mathbf{I}) \frac{1}{r^{3}},
$$

where $g_{\mathrm{n}}$ is the nuclear $g$-factor, $\alpha$ is the fine structure constant, $m_{\mathrm{p}}$ is the proton mass and $\vec{\alpha}$ is the vector of Dirac matrices. This operator is known to be almost diagonal in quantum number $l$ and in the following calculations we neglect nondiagonal terms.

Let us start with the nonrelativistic expressions for a spherical wave $l \neq 0$. It is not difficult to derive, that for the $\sigma$-type state $|l, 0\rangle$

$$
A=0, \quad A_{\mathrm{d}}=\frac{g_{\mathrm{n}} \alpha^{2}}{2 m_{\mathrm{p}}} \frac{l(l+1)}{(2 l-1)(2 l+3)}\left\langle n, l\left|\frac{1}{r^{3}}\right| n, l\right\rangle .
$$

So, in the nonrelativistic approximation only s-wave contributes to the isotropic constant $A$. Note that the radial integral in (11) is rapidly decreasing with $l$, while the coefficient in front of it is a weak function of $l$.

Interaction of the EDM of the electron with the molecular electric field $-\nabla \phi$ is also singular at the nucleus [15]. The most convenient form of this operator is [16:

$$
H_{d}=2 d_{\mathrm{e}}\left(\begin{array}{cc}
0 & 0 \\
0 & \vec{\sigma}
\end{array}\right)(-\nabla \phi)
$$

Relativistic expressions for $A, A_{\mathrm{d}}$ and $W_{d}$ can be found in [3,4,1. In the paper [4] these constants were calculated for the wave function (9) with $c_{\mathrm{p}}=\delta_{\mathrm{f}}=c_{\mathrm{f}}=0$ :

$$
\begin{aligned}
A & =\left(11850 x_{\mathrm{s}}^{2}-102 x_{\mathrm{p}}^{2}-5 x_{\mathrm{d}}^{2}+\cdots\right) \mathrm{MHz} \\
A_{\mathrm{d}} & =\left(422 x_{\mathrm{p}}^{2}+36 x_{\mathrm{d}}^{2}+\cdots\right) \mathrm{MHz} \\
W_{d} & =\left(-29.7 x_{\mathrm{s}} x_{\mathrm{p}}+1.3 x_{\mathrm{p}} x_{\mathrm{d}}+\cdots\right) \text { a.u. }
\end{aligned}
$$


If we neglect the $\mathrm{d}$-wave terms and use the experimental values (2) for $A$ and $A_{\mathrm{d}}$, we can solve (13) - (15) for $x_{\mathrm{s}}, x_{\mathrm{p}}$ and $W_{d}$ :

$$
\begin{array}{r}
x_{\mathrm{s}}=0.803, \quad x_{\mathrm{p}}=0.492, \\
W_{d}=-11.7 \text { a.u. }=-1.45 \cdot 10^{25} \mathrm{~Hz} /(\mathrm{e} \mathrm{cm}),
\end{array}
$$

where sings of $x_{\mathrm{s}}$ and $x_{\mathrm{p}}$ are chosen to account for the repulsion of the unpaired electron from the $\mathrm{F}^{-}$ion.

Result (17) is more accurate than one can expect from (13) - (15). It follows from the proportionality between $W_{d}$ and $\sqrt{A A_{\mathrm{d}} \text { : }}$

$$
W_{d}=-1.69 \cdot 10^{16} \sqrt{A A_{\mathrm{d}}} 1 /(\mathrm{e} \mathrm{cm})
$$

which is based on the behaviour of s- and p-waves in the vicinity of the nucleus where molecular potential is close to that of the nucleus. In contrast to that, results (16) depend on the assumption that spherical waves in (5) correspond to the orbitals of $\mathrm{Yb}^{+}$.

Below we calculate several corrections to (17) and (18), which mostly account for contribution of the higher spherical waves. If we use the normalization condition $x_{\mathrm{s}}^{2}+x_{\mathrm{p}}^{2}+x_{\mathrm{d}}^{2} \approx 1$, we can solve equations $(13)-(15)$ with the $\mathrm{d}$-wave included:

$$
x_{\mathrm{s}}=0.803 ; \quad x_{\mathrm{p}}=0.481, \quad x_{\mathrm{d}}=0.356, \quad W_{d}=-11.2 \text { a.u. }
$$

Comparison of (17) and (19) gives the following correction coefficient for the d-wave contribution to $W_{d}$ :

$$
k_{\mathrm{d}}=0.96
$$

Note that the normalization condition give the upper bound for the coefficient $x_{\mathrm{d}}$. So, it is likely, that (19) and (20) somewhat overestimate the d-wave contribution.

\section{Spin-doubling constant}

Let us estimate the mixing coefficient $c_{\mathrm{p}}$ in $(9)$ :

$$
c_{\mathrm{p}}=\frac{\left\langle\Pi\left(A_{1}\right)\left|H_{\mathrm{so}}\right| \Sigma(X)\right\rangle}{E_{X}-E_{A}}=\frac{\left\langle\pi_{\mathrm{p}}, \omega\left|H_{\mathrm{so}}\right| \sigma_{\mathrm{s}}, \omega\right\rangle}{E_{X}-E_{A}} \approx \frac{x_{\mathrm{p}} a_{\mathrm{p}} \xi_{6,1}}{\sqrt{2}\left(E_{X}-E_{A}\right)},
$$

where we use (5) and (7) to calculate the numerator:

$$
\begin{gathered}
\left\langle\pi_{\mathrm{p}}, \omega\left|H_{\mathrm{so}}\right| \sigma_{\mathrm{s}}, \omega\right\rangle \approx x_{\mathrm{p}} a_{\mathrm{p}}\left\langle\tilde{6} p_{1},-\frac{1}{2}\left|H_{\mathrm{so}}\right| \tilde{6} p_{0}, \frac{1}{2}\right\rangle \approx x_{\mathrm{p}} a_{\mathrm{p}}\left\langle 6 p_{1},-\frac{1}{2}\left|H_{\mathrm{so}}\right| 6 p_{0}, \frac{1}{2}\right\rangle, \\
\left\langle n, l, 1,-\frac{1}{2}\left|H_{\mathrm{so}}\right| n, l, 0, \frac{1}{2}\right\rangle \equiv-\xi_{n, l}\left\langle l, 1\left|l_{1}\right| l, 0\right\rangle\left\langle-\frac{1}{2}\left|s_{-1}\right| \frac{1}{2}\right\rangle=\frac{1}{2} \xi_{n, l} \sqrt{l(l+1)}
\end{gathered}
$$

and $\xi_{n, l}$ is the atomic spin-orbit constant for the $(n, l)$-shell. It is proportional to the radial integral which enters (11). For the $\mathrm{Yb}^{+}$ion $\xi_{6,1}=1900 \mathrm{~cm}^{-1}$.

The level (17) is identified as $A_{1}\left(E_{A_{1}}=18090 \mathrm{~cm}^{-1}\right)$. It's fine splitting with the level $\mathrm{A}_{2}\left(\Pi_{3 / 2}\right)$ is $1370 \mathrm{~cm}^{-1}[10]$. Within the same approximation we can link this splitting to the constant $\xi_{6,1}$ and find parameter $a_{\mathrm{p}}$ :

$$
E_{A_{2}}-E_{A_{1}} \approx a_{\mathrm{p}}^{2} \xi_{6,1}, \quad \Rightarrow \quad a_{\mathrm{p}}^{2} \approx 0.72
$$

In a same manner we can calculate coefficient $c_{\mathrm{f}}$ in $(9)$. In this case the spin-orbit interaction mixes $\sigma_{\mathrm{f}}$-hole with $\pi_{\mathrm{f}}$-hole:

$$
c_{\mathrm{f}}=\frac{\left\langle\Pi(F)\left|H_{\mathrm{so}}\right| \Sigma(X)\right\rangle}{E_{X}-E_{F}} \approx \delta_{\mathrm{f}} \frac{\overline{\left\langle\pi_{\mathrm{f}},-\omega\right|} H_{\mathrm{so}} \overline{\left|\sigma_{\mathrm{f}},-\omega\right\rangle}}{E_{X}-E_{F}} \approx \frac{-\sqrt{3} \delta_{\mathrm{f}} \bar{\xi}_{4,3}}{E_{X}-E_{F}} .
$$

The only problem here is that molecular state with the $\pi_{\mathrm{f}}$-hole, which we define as $\Pi(F)$, is not known, and thus we do not know the energy denominator. The spin-orbit constant for the f-hole has the opposite to normal sign: $\bar{\xi}_{4,3}=-3665 \mathrm{~cm}^{-1}$.

Formulae (21), (24) and (25) reduce the number of independent parameters in the wave function (9). Still, it has two extra parameters $\delta_{\mathrm{f}}$ and $\varepsilon_{\mathrm{d}}$ as compared to the wave function (5) which was used in (13) - (15). Below we eliminate parameter $\delta_{\mathrm{f}}$ using experimental value of the spin-doubling constant $\gamma$. 
The spin-doubling term in the effective Hamiltonian (11) arise from the spin-orbit interaction. It was shown in [9] that

$$
\gamma=2 B\left(1-\left\langle\Sigma(X), \frac{1}{2}\left|J_{e,+}\right| \Sigma(X),-\frac{1}{2}\right\rangle\right)
$$

where $\mathbf{J}_{e}=\mathbf{L}+\mathbf{S}$ is the total angular momentum of the electrons. For the pure $\Sigma$ state $\left\langle\mathbf{J}_{e}\right\rangle=\langle\mathbf{S}\rangle$ and $(26)$ gives $\gamma=0$. This is no longer true, when the spin-orbit corrections are taken into account. With the help of (26), (21) and (25) it is easy to calculate $\gamma$ for the state (9):

$$
\gamma=2 B\left(2\left(x_{\mathrm{p}} a_{\mathrm{p}}\right)^{2} \frac{\xi_{6,1}}{E_{X}-E_{A}}+12 \delta_{\mathrm{f}}^{2} \frac{\bar{\xi}_{4,3}}{E_{X}-E_{F}}\right) .
$$

Note that numerical factors in parentheses are equal to $l(l+1)$.

As we already pointed out, the second denominator in (27) is unknown. If we use corresponding energy interval for the $\mathrm{Yb}^{+}$ion, we receive the following relation between $x_{\mathrm{p}}$ and $\delta_{\mathrm{f}}$

$$
2100 x_{\mathrm{p}}^{2}-11150 \delta_{\mathrm{f}}^{2}=13, \quad \Rightarrow \quad \delta_{\mathrm{f}}^{2} \approx 0.19 x_{\mathrm{p}}^{2}
$$

This equation shows that experimental value of $\gamma$ correspond to almost complete cancelation between p-wave and f-wave contributions. To obtain this relation between $\delta_{\mathrm{f}}$ and $x_{\mathrm{p}}$ we used ionic spin-orbit constants $\xi_{n, l}$ and ionic denominator in (27). So, we can not expect it to be much better than an order of magnitude estimate.

\section{Spin-orbit and f-hole corrections to $W_{d}$}

It follows from (11), (27) and (28), that for the molecular state (9) the f-wave contribution to the constant $A_{\mathrm{d}}$ should be approximately 6 times smaller than that of the p-wave. Indeed, the two contributions to the constant $\gamma$ cancel each other, but in (27) there is the factor $l(l+1)$ which is not present in (11).

A straightforward relativistic calculation result in the following f-hole correction to the hyperfine tensor

$$
\begin{aligned}
\delta A & =-\frac{g_{\mathrm{n}} \alpha}{2 m_{\mathrm{p}}} \delta_{\mathrm{f}}^{2}\left(\frac{12}{49} h_{5 / 2,5 / 2}+\frac{32}{49} h_{5 / 2,7 / 2}+\frac{64}{147} h_{7 / 2,7 / 2}\right) \text { a.u., } \\
\delta A_{\mathrm{d}} & =\frac{g_{\mathrm{n}} \alpha}{2 m_{\mathrm{p}}} \delta_{\mathrm{f}}^{2}\left(\frac{48}{245} h_{5 / 2,5 / 2}-\frac{8}{49} h_{5 / 2,7 / 2}+\frac{64}{441} h_{7 / 2,7 / 2}\right) \text { a.u. }
\end{aligned}
$$

The radial integrals $h_{j, j^{\prime}}$ here have the form $h_{j, j^{\prime}}=\int_{0}^{\infty} \mathrm{d} r\left(f_{j} g_{j^{\prime}}+g_{j} f_{j^{\prime}}\right)$, where $f_{j}$ and $g_{j}$ are the upper and lower components of the radial Dirac wave function. Again we take radial integrals for the $\mathrm{Yb}^{+}$ion and use (28) to arrive at

$$
\delta A=-11 x_{\mathrm{p}}^{2} \mathrm{MHz}, \quad \delta A_{\mathrm{d}}=64 x_{\mathrm{p}}^{2} \mathrm{MHz} .
$$

It is clear, that correction (31) to isotropic constant $A$ (13) is negligible, while correction to the dipole constant $A_{\mathrm{d}}$ (14) is about $15 \%$ for a given $x_{\mathrm{p}}$, which is in a good agreement with our nonrelativistic estimate $\frac{1}{6}$.

With the f-hole correction (31) included, equation (14) is changed to

$$
A_{\mathrm{d}}=\left(486 x_{\mathrm{p}}^{2}+36 x_{\mathrm{d}}^{2}\right) \mathrm{MHz}
$$

and we obtain the following f-hole correction coefficient to $W_{d}$

$$
k_{\mathrm{f}}=0.93
$$

In deriving (33) we have ignored the direct f-wave contribution to the constant $W_{d}$ (see (15)). Such contribution is proportional to a small product $x_{\mathrm{d}} \delta_{\mathrm{f}} \leq 0.1$ and is less than $1 \%$.

The last correction to the constant $W_{d}$ is associated with the admixture of the $\pi_{\mathrm{p}}$ state to (9). According to (21) $c_{\mathrm{p}} \approx-0.06 x_{\mathrm{p}}$. This admixture changes weights of the $\mathrm{p}_{1 / 2^{-}}$and $\mathrm{p}_{3 / 2}$-waves in the wave function. That, in turn, slightly changes coefficients in (14) and (15) (note, that only $\mathrm{p}_{1 / 2}$-wave contributes to $W_{d}$ ). A simple calculation give

$$
k_{\mathrm{so}}=0.98 \text {. }
$$




\section{Discussion}

Taking into account (20), (33) and (34), we obtain the total correction factor for $W_{d}$ to be $k_{\text {corr }}=k_{\mathrm{d}} k_{\mathrm{f}} k_{\text {so }}=0.87$. Applying it to (18), we arrive at

$$
W_{d}=-10.2 \text { a.u. }=-1.26 \cdot 10^{25} \mathrm{~Hz} /(\mathrm{e} \mathrm{cm})
$$

We have calculated corrections to the semiempirical value of the constant $W_{d}$ of the spin-rotational Hamiltonian (11) which can account for the $P, T$-odd effects in $\mathrm{YbF}$ molecule. The main correction is caused by the f-hole admixture to the ground state. Two other corrections account for the spin-orbit interaction and for the d-wave term in the wave function. Altogether they reduce the answer by more than $10 \%$.

We think that wave function (9) includes two most important configurations. Admixture of other configurations will tend to decrease constant $W_{d}$, so we can expect that our value (35) is slightly overestimated. But it is unlikely, that corresponding corrections are significantly larger than those discussed above. So, we estimate the accuracy of our calculation to be about $20 \%$, which is typical to the semiempirical method.

$A b$ initio calculation [13] gave smaller absolute value for $W_{d}$, but it also underestimated both $A$ and $A_{\mathrm{d}}$. It is more informative to compare the coefficient in the equation (18) which correspond to different calculations. Our final value for this coefficient is $-1.47 \cdot 10^{16} 1 /(\mathrm{e} \mathrm{cm})$, while results of [13] correspond to $-1.75 \cdot 10^{16} 1 /(\mathrm{e} \mathrm{cm})$. So, in this sense, our result is even smaller than that of [13].

In this paper we have not considered other possible sources of $P, T$-violation, such as scalar neutral currents 7 and magnetic quadrupole moment of the nucleus 17. Corresponding constants $W_{\mathrm{S}}$ and $W_{\mathrm{M}}$ of the spin-rotational Hamiltonian can be calculated in a similar way to $W_{d}$. 4 . Our value for the former constant is: $W_{\mathrm{S}}=-43 \mathrm{kHz}$. Because of the higher multipolarity of the electronic operator associated with the constant $W_{\mathrm{M}}$, this interaction is much more sensitive to the higher terms of the spherical wave expansion of the molecular wave function. Thus, it is more difficult to make accurate calculation of this interaction within the semiempirical approach.

\section{Acknowledgments}

The author is grateful to E A Hinds, I B Khriplovich, H M Quiney and A V Titov for valuable discussions.

[1] Kozlov M G and Labzowsky L N 1995 J. Phys. B 281933

[2] Sauer B E, Peck S K, Redgrave G and Hinds E A 1996 Private communication

[3] Kozlov M G 1985 JETP 621114

[4] Kozlov M G and Ezhov V F 1994 Phys. Rev. A 494502

[5] Khriplovich I B 1997 Private communication; quoted in CP-Violation Without Strangeness, (Springer, in press)

[6] Sushkov O P, Flambaum V V 1978 JETP 48608

[7] Gorshkov V G, Labzowsky L N and Moskalev A N 1979 ZhETF 76414 [JETP 49]

[8] Flambaum V V and Khriplovich I B 1985 Phys. Lett. 110A 12

[9] Kozlov M G, Labzowsky L N and Mitruschenkov A O 1991 JETP 73415

[10] Huber K P, Herzberg G 1979 Molecular Spectra and Molecula Structure. IV. Constants of Diatomic Molecules (Van Nostrand, New York)

[11] Sauer B, Wang J, and Hinds E A 1995 Phys. Rev. Lett. 741554

[12] Knight L B, Jr. and Weltner W, Jr. 1970 J. of Chem. Phys. 534111

[13] Titov A V, Mosiagin N S, Ezhov V F 1996 Phys. Rev. Lett. 775346

[14] Quiney H M, Skaane H and Grant I P 1997 submitted to Adv. Quant. Chem.

[15] Sandars P G H 1965 Phys. Lett. 14 194; 196622290

[16] Khriplovich I B 1991 Parity Non-Conservation in Atomic Phenomena (Gordon and Breach, New York)

[17] Sushkov O P, Flambaum V V and Khriplovich I B 1984 JETP 60873 